\title{
A comparative study of pregnancy outcome of sequential versus day 3 versus only blastocyst (day 6) transfer at a single IVF center over one year
}

\author{
Purnima Kishore Nadkarni ${ }^{1}$, Kishore Mohan Nadkarni ${ }^{1}$, Pooja Prabhakar Singh ${ }^{2}$, \\ Prabhakar Singh ${ }^{2}$, Aditi Nadkarni ${ }^{1}$, Kanika Rajendra Kalyani ${ }^{1}$, Rinoy Shreedharan ${ }^{3}$
}

\author{
${ }^{1}$ Nadkarni Hospital and Test Tube Baby Centre, Killa, Pardi, Vapi, Gujarat, India \\ ${ }^{2} 21$ st Century Hospital, Surat, Gujarat, India \\ ${ }^{3}$ Krishna Hospital, Korba, India
}

Received: 13 June 2015

Revised: 21 June 2015

Accepted: 10 July 2015

\section{*Correspondence:}

Dr. Kanika. R. Kalyani,

E-mail: kanika.kalyani@gmail.com

Copyright: (c) the author(s), publisher and licensee Medip Academy. This is an open-access article distributed under the terms of the Creative Commons Attribution Non-Commercial License, which permits unrestricted non-commercial use, distribution, and reproduction in any medium, provided the original work is properly cited.

\begin{abstract}
Background: The recent introduction of sequential media has refocused attention upon the role of human blastocyst in IVF. For optimisation of extended cycles, one needs to give importance to all the aspects of treatment cycle like the stimulation regimens, medium composition, endometrial quality and uterine receptivity. This study was done to know the pregnancy outcomes of Day 3 transfer vs Day3/Day 6 transfer vs only Day 6 transfer.

Methods: It was a retrospective study in which 342 patients undergoing ICSI were included. 199 women underwent Day 3/Day 6 sequential transfers, 112 underwent only Day 3 transfer and only 30 women were given only Blastocyst i.e. day 6 transfer. Pregnancy outcomes of all the three groups were studied.

Results: Sequential transfer was found to give maximum pregnancy rate and highest implantation rate. But it was also associated with highest number of multiple pregnancies.

Conclusions: Sequential transfer is a very good efficacious approach in ART cycles if extended media are available as it gave maximum pregnancy rate and implantation rate. However it is associated with multiple pregnancies. Thus the ultimate goal is to have a single blastocyst transfer with better outcomes and lower multiple pregnancy rates.
\end{abstract}

Keywords: Day 3 transfer, Sequential transfer, Blastocyst transfer, Implantation rate

\section{INTRODUCTION}

The first clinical human pregnancy utilizing in vitro fertilization was established by the transfer of a blastocyst. ${ }^{1}$ However, due to difficulties in maintaining the human embryo in culture for more than a couple of days, it became a routine to do cleavage-stage transfers. The recent introduction of sequential media has refocused attention upon the role of human blastocyst in IVF. For optimisation of extended cycles, one needs to give importance to all the aspects of treatment cycle like the stimulation regimens, medium composition, endometrial quality and uterine receptivity. In all mammalian studies, it has been found that the post compaction mammalian embryo can tolerate a wider range of environments. By placing the human embryo into the uterus before compaction it will expose the embryo to concentrations of carbohydrates ${ }^{2}$ and amino acids ${ }^{3,4}$ which, it is not normally exposed to. Thus the cleavage stage human embryo will have to adapt its physiology and metabolism in response to the uterine environment. It is evident from 
in vitro studies that such adaptation to its environment gives a lot of stress to the embryo. Also, because of ovarian hyperstimulation, the uterine milieu is compromised ${ }^{5}$. So therefore it is better to expose embryos to such an altered environment for as short a period as possible and this can be facilitated by blastocyst transfer. Recently, Fanchin and colleagues have observed that uterine junctional zone contractions progressively decrease as one moves farther into the luteal phase, and that fewer uterine contractions are associated with improved pregnancy rates indicating that the early transfer of embryos to the uterus may lead to embryo loss. Also, recently, the ability to support human embryo in culture has improved a lot. Special blastocyst media are now available that can support high levels of human blastocyst development which can subsequently implant at very high rates (around 50\%). ${ }^{6}$ Another application for blastocyst culture is to allow for preimplantation genetic diagnosis (PGD) or pre-implantation genetic screening (PGS). But, inspite of all these advances, current evidence concerning the advantages of day $5 / 6$ blastocyst stage embryo transfer (ET) over day $2 / 3$ cleavage-stage ET is controversial (Levron et al, 2002; Phillips et al, 2003; Blake et al., 2004). Furthermore, cultured embryos occasionally fail to reach the blastocyst stage resulting in cancellation of the entire treatment cycle with negative emotional and economic consequences for the patient (Ashkenazi et al., 2000). Sequential transfer of day 3 and day 5/6 embryos may offer an alternative approach that takes advantage of the cumulative success of both conventional cleavage-stage and blastocyst-stage ET (Goto et al, 2003). This approach confers the supposed benefits of blastocyst transfer without endangering the cycle owing to failure of embryos to survive the prolonged culture. The efficacy of this procedure, however, is still a matter of debate. The purpose of this study was to evaluate the effect of sequential embryo transfer i.e. day3/day6, only blastocyst transfer and only Day 3 embryo transfer in ART cycles at Killa Pardi, Gujarat. The clinical pregnancy rate following IVFembryo transfer is usually $40-50 \%{ }^{7}$

\section{METHODS}

It is a retrospective comparative study. 342 women undergoing ICSI at Nadkarni Hospital and Test Tube Baby Centre, Killa Pardi were studied. 199 patients underwent sequential transfer of day 3 embryos and day 5/6 blastocysts, 112 subjects had undergone day 3 embryo transfer only and 30 subjects underwent only day 6 blastocyst transfer. All the groups were matched according to age, cause of infertility and basal FSH levels. Ovaries were stimulated and oocytes retrieved according to conventional IVF protocols in which midluteal gonadotrophin releasing hormone $(\mathrm{GnRH})$ agonist administration is followed by ovarian stimulation by gonadotrophins (Dor et al, 1997) or gonadotrophins from day 2 with $\mathrm{GnRH}$ ant from day 7/8. The stimulation protocols were similar throughout the study period. In the sequential group, 2 of the best cleaved embryos were transferred on day 3 followed by one blastocyst on day 6 . In day 3 transfer group, only 3 embryos were transferred on day 3 and only blastocyst was transferred on day 6 in the blastocyst group. A commercial sequential IVF medium was used for all gamete and embryo handling procedures. For extended embryo culture, day 3 embryos were transferred into 80 droplets of BLT medium under oil. On day 6, blastocysts were selected for replacement and cryopreservation according to a morphological assessment of inner cell mass and extent of cavity expansion, in addition to the estimated number of trophectodermal cells. The number of embryos available and their morphology, as well as maternal age, IVF history, and number of prior implantation failures, were taken into account when deciding on the number of embryos for replacement. A soft transfer catheter (cook) was used for both ET procedures. In rare cases of multiple IVF failures of unknown cause, or when embryo quality was poor, more than four embryos were transferred after detailed discussion with the couple concerning the risks of multiple pregnancies. The implantation rate in each group was defined as the total number of gestational sacs (observed by ultrasound) divided by the total number of embryos/blastocysts transferred. Clinical pregnancy rates were calculated as the number of ultrasound tests showing heartbeat at 6 weeks of pregnancy divided by the number of women in the group. Also recorded were the numbers of multiple pregnancies, and pregnancy outcome.

\section{RESULTS}

Total 341 patients were studied. Maximum i.e. 199(58\%) subjects underwent sequential transfer, 112(42\%) subjects underwent only day 3 transfer and only 30 patients were just given an only Blastocyst i.e. day 6 transfer. Age was uniformly distributed amongst all the three groups with the day 3 group subjects having an average age of $31.05 \pm 5.33$ years, sequential group subjects having a mean age of $31.49 \pm 5.57$ years and the Day 6 i.e. Blastocyst group had an average age of $31.17 \pm 5.41$ years. There was no significant difference between the basal FSH values amongst subjects of all the three groups. The sequential group had the maximum number of retrieved oocytes i.e. $13.30 \pm 6.41$ which was why we could transfer 3 embryos for that group ( 2 on day 3 and 1 on day 6). The day 3 group had 9.92 \pm 5.97 eggs retrieved and day 6 transfer group had 10.61 17.29 eggs. Similarly the sequential group had maximum number of eggs fertilized $(8.70 \pm 13.70)$ which was statistically significant ( $F$ value 5.18, p-value 0.006). Number of eggs cleaved were also maximum in the sequential transfer group which was also statistically significant ( $\mathrm{F}$ value 3.55 , p-value 0.030) followed by the Day 3 group and the day 6 group. Grade 1 blastocysts were maximum in the sequential transfer group with a high $F$ value of 18.74 and thus a high statistical signicance ( $\mathrm{p}$-value 0.000). Average Beta HCG value on Day 15 counting from the day of ovum pick up and ICSI was 702.12 \pm 951.43 in the Sequential group, $440.57 \pm 732.22$ in the day 3 groups and 
$307.42 \pm 595.66$ in the Day 6 group. One of the disadvantages of sequential transfer was the number of multiple pregnancies [134(67.34\%)]. But since we practice IVF at a rural set up, patients cannot afford multiple cycles of IVF and if they don't conceive once, they fail to follow up because of lack of funds. This is why our result oriented approach and cost effective ART practice coaxed us to transfer 3 embryos which led to a

Table 1: Demographic data.

\begin{tabular}{|lccccc|} 
& Day 3 & Sequential & Only Blasto & F-value & p-value \\
\hline No. of women & 112 & 199 & 30 & - & - \\
\hline Age & $31.05 \pm 5.33$ & $31.49 \pm 5.57$ & $31.17 \pm 5.41$ & 0.24 & $0.78, \mathbf{N S}$ \\
\hline Retrieved oocytes & $9.92 \pm 5.97$ & $13.30 \pm 6.41$ & $10.61 \pm 7.29$ & 9.42 & $0.000, \mathbf{S}$ \\
\hline No. of eggs fertilized & $4.28 \pm 2.98$ & $8.70 \pm 13.70$ & $4.94 \pm 4.71$ & 5.18 & $0.006, \mathbf{S}$ \\
\hline No. of eggs cleaved & $4.37 \pm 3.13$ & $8.93 \pm 15.28$ & $4.16 \pm 4.75$ & 3.55 & $0.030, \mathbf{S}$ \\
\hline Grade 1 blasto & $3(2.68 \%)$ & $146(73.37 \%)$ & $11(36.67 \%)$ & 18.74 & $0.000, \mathbf{S}$ \\
\hline $\begin{array}{l}\text { (BHCG values) } \\
\text { Pregnancy rate }\end{array}$ & $440.57 \pm 732.22$ & $702.12 \pm 951.43$ & $307.42 \pm 595.66$ & 3.48 & $0.032, \mathbf{S}$ \\
\hline $\begin{array}{l}\text { Multiple pregnancies per } \\
\text { pregnancy (No. of multiple } \\
\text { pregnancies/ total number of } \\
\text { positive pregnancies) }\end{array}$ & $52(46.43 \%)$ & $134(67.34 \%)$ & $8(26.67 \%)$ & 25.02 & $0.000, \mathbf{S}$ \\
\hline $\begin{array}{l}\text { No. of multiple pregnancies } \\
\text { Implantation rate }\end{array}$ & $39(34.82 \%)$ & $85(42.71 \%)$ & $4(13.33 \%)$ & 2.10 & $0.23, \mathbf{N S}$ \\
\hline & $0.28 \pm 0.34(28 \%)$ & $0.39 \pm 0.36(39 \%)$ & $0.30 \pm 0.05$ & 7.56 & $0.001, \mathbf{S}$ \\
\hline
\end{tabular}

slightly higher multiple pregnancy rate. It was much easier to have a good obstetric set up and provide better antenatal care to these high risk pregnancies and taking them upto term than dealing with a relatively lower pregnancy rate with single embryo transfers which created a major dissatisfaction. Multiple pregnancy rate was $52(46.43 \%)$ in day 3 transfers and $8(26.67 \%)$ in the day 6 transfer group. Implantation rate was the maximum in sequential group $39 \%$ which was statistically significant. Thus sequential transfer not only increased pregnancy rates but also implantation rates.

\section{DISCUSSION}

Two prospective randomized trials using sequential media for blastocyst culture have been reported. ${ }^{8,9}$ In the first trial the selection criteria included patients having more than 10 follicles at the time of HCG, while in the second trial the selection criterion was patients having four or more fertilized oocytes. In the study of Glujovsky et al, ${ }^{8}$ an implantation rate of $50.5 \%$ (FHB) was attained in the day 5 group, compared to $30.1 \%$ in the control (day 3 transfer). Our study had an implantation rate of $30 \%$ in the day 5 group and $28 \%$ in the day 3 groups which was also statistically significant. In contrast, in the study of Coskun et al, there was no significant increase in implantation rate when embryos were transferred on day 5 rather than on day $3 .{ }^{9}$ However, Coskun et al., found that even in the best prognosis patients, i.e. those under 30 years of age or those with more than 5 good embryos on day 3 , an implantation rate of $30 \%$ was never attained in the day 5 group. ${ }^{9}$ But because several other studies were talking in favor of blastocysts as regards implantation rates, maybe many other factors affected the study of Coskum et al. Shapiro et al., and Langley et al., both reported that human blastocysts can be formed from embryos with only a few cells on day 3 and they can subsequently implant. ${ }^{10}$ Racowsky et al. reported different results. Using media in sequence for extended culture they found no cases of failed blastocyst development when there were three or more 8-cell day-3 embryos available for culture.

Clinical trials of blastocyst culture and transfer have largely focused on patients with a good prognosis, with an adequate response to gonadotropins. However, in a retrospective study, in which all patients attending a fertility clinic underwent blastocyst culture and transfer, it was determined that extended culture resulted in increased implantation and pregnancy rates, compared to the use of cleavage-stage embryo transfer. Implantation rates after extended embryo culture were $32.4 \%$, significantly higher than that obtained after the transfer of cleavage-stage embryos on day $3(23.3 \%)$. Although there was a significant increase in the percentage of patients who did not have an embryo transfer $(6.7 \%$, compared to patients having a day 3 transfer at $2.9 \%$ ), pregnancy rates per oocyte retrieval were higher for day 5 transfers $(57.5 \%)$ compared to day 3 transfers $(46.1 \%)$. Furthermore, the number of embryos transferred on day 5 (2.5) was significantly below that transferred on day 3 (3.0). Some studies that observed a higher implantation 
for transferred blastocysts also have reported a high rate of twinning $(53 \%)$ despite transfer of only two blastocysts. ${ }^{8}$ This was similar to our study where we found $67.34 \%$ multiple pregnancy rate in sequential transfer which was statistically significant.

\section{CONCLUSIONS}

Sequential transfer is an efficacious approach as regards achieving a higher implantation rate and pregnancy rate. One major disadvantage with sequential transfer is the increased incidence of multiple pregnancies. Blastocyst culture and transfer represents an effective means of eliminating high order multiple gestations in good prognosis patients, and more recently it has been shown that it can be applied to all patients entering an IVF program with a concomitant increase in the efficiency of patient treatment. Certainly blastocyst transfer, with resultant high implantation rates, should be considered for patients electing to have a single embryo transferred. However, there are those who believe that for some, if not all patients, embryos would be better off being replaced in the uterus on day 3. Time will tell which approach is correct.

In programs where a comparison of day 3 vs. day 5 transfers has been made the majority report a benefit of moving to blastocyst culture. The time has come to focus on oocyte quality and endometrial receptivity along with culture conditions. Before extended culture is considered, all aspects of clinical and laboratory procedures need to be optimized. Should problems exist either in patient stimulation protocols or within the laboratory, extended culture may only exacerbate the situation. Extended culture should first be tried on oocyte donors, or those patients who respond well to gonadotropins. Implantation rates of $40 \%$ or greater should readily be obtained in such patients. Though sequential transfer offers a good outcome in ART cycles, our ultimate goal is to have a single blastocyst transfer yielding higher implantation rates and lower incidence of multiple pregnancies.

Funding: No funding sources

Conflict of interest: None declared
Ethical approval: The study was approved by the Institutional Ethics Committee

\section{REFERENCES}

1. Steptoe PC, Edwards RG. Birth after the reimplantation of a human embryo. Lancet. 1978;2(8085):366.

2. Gardner DK, Lane M, Calderon I, Leeton J. Environment of the preimplantation human embryo in vivo: metabolite analysis of oviduct and uterine fluids and metabolism of cumulus cells. Fertil Steril. 1996;65(2):349-53.

3. Iritani A, Nishikawa Y, Gomes WR, Van Demark NL. Secretion Rates and Chemical Composition of Oviduct and Uterine Fluids in Rabbits. J Anim Sci. 1971;33:829-35.

4. Miller JG, Schultz GA. Amino acid content of preimplantation rabbit embryos and fluids of the reproductive tract. Biol Reprod. 1987;36:125-9.

5. Simon C, Garcia Velasco JJ, Valbuena D, Peinado $\mathrm{JA}$, Moreno C, Remohi J, et al. Increasing uterine receptivity by decreasing estradiol levels during the preimplantation period in high responders with the use of a follicle-stimulating hormone step-down regimen. Fertil Steril. 1998;70:234-9.

6. Gardner DK, Lane M. Culture of viable human blastocysts in defined sequential serum -free media. Hum Reprod. 1998;13(3):148-59.

7. Margalioth EJ, Ben- Chetrit A, Gal M, Eldar-Geva T. Investigation and treatment of repeated implantation failure following IVF-ET. Hum Reprod 2006;21:3036-43.

8. Glujovsky D, Blake D, Farquhar C, Bardach A. Cleavage stage versus blastocyst stage embryo transfer in assisted reproductive technology. Cochrane Database Syst Rev. 2012;11;7:CD002118.

9. Coskun S, Hollanders J, Al-Hassan S, Al-Sufyan H, Al-Mayman H, Jaroudi K. Day 5 versus day 3 embryo transfer: a controlled randomized trial. Hum Reprod. 2000;15(9):1947-52.

10. Langley MT, Marek DM, Gardner DK, Doody KM, Doody KJ. Extended Embryo culture in human assisted reproduction treatments. Hum Reprod. 2001;16(5):902-8.

Cite this article as: Nadkarni PK, Nadkarni KM, Singh PP, Singh P, Nadkarni A, Kalyani KR, Shreedharan R. A comparative study of pregnancy outcome of sequential versus day 3 versus only blastocyst (day 6) transfer at a single IVF center over one year. Int J Reprod Contracept Obstet Gynecol 2015;4:2032-5. 